\title{
Relationship Between Door-to-Embolization Time and Clinical Outcomes After Transarterial Embolization in Trauma Patients With Severe Pelvic Fracture
}

\section{Hohyun Kim}

Pusan National University Hospital

\section{Chang Ho Jeon}

Pusan National University Hospital

Jae Hun Kim ( $\nabla$ drtrauma73@gmail.com )

Pusan National University Hospital https://orcid.org/0000-0003-4504-9898

\section{Hoon Kwon}

Pusan National University Hospital

\section{Chang Won Kim}

Pusan National University Hospital

\section{Gil Hwan Kim}

Pusan National University Hospital

\section{Chan Kyu Lee}

Pusan National University Hospital

\section{Sang Bong Lee}

Pusan National University Hospital

Jae Hoon Jang

Pusan National University Hospital

\section{Seon Hee Kim}

Pusan National University Hospital

\section{Seok Ran Yeom}

Pusan National University Hospital

\section{Research article}

Keywords: multiple trauma, therapeutic embolization, pelvic bone, mortality rate, time

Posted Date: May 21st, 2020

DOl: https://doi.org/10.21203/rs.3.rs-29651/v1

License: (c) (i) This work is licensed under a Creative Commons Attribution 4.0 International License. Read Full License 


\section{Abstract}

Background: While transarterial embolization (TAE) is an effective way to control arterial bleeding associated with pelvic fracture, the clinical outcomes according to door-to-embolization (DTE) time are unclear. This study investigated how DTE time affects outcomes in patients with severe pelvic fracture.

Methods: Using a trauma database between November 1, 2015 and December 31, 2019, trauma patients undergoing TAE were retrospectively reviewed. The final study population included 204 patients treated with TAE. The relationships between DTE time and patients' outcomes were evaluated. Multivariate binomial logistic regression analyses, multivariate linear regression analyses, and multivariate Cox hazard proportional regression analyses were performed to estimate the impacts of DTE time on clinical outcomes.

Results: The median DTE time was 150 min (interquartile range, 123-186). The mortality rates at 7 and 28 days and overall were $8.3 \%, 13.7 \%$, and $15.7 \%$, respectively. DTE time served as an independent risk factor for mortality at 7 and 28 days (adjusted odds ratio $=1.62,95 \%$ confidence interval $[\mathrm{Cl}]=1.14-2.30, p=0.007$; adjusted odds ratio $=1.48, \mathrm{Cl}=$ 1.05-2.07, $p=0.023$, respectively). In multivariate Cox proportional hazards regression analyses, the adjusted hazard ratio of DTE time for mortality at 28 days was $1.28(\mathrm{Cl}=1.08-1.30, p=0.005)$. In addition, there was a positive relationship between DTE time and requirement for packed red blood cell transfusion during the initial $24 \mathrm{~h}$ and a negative relationship between DTE time and ICU-free days to day 28.

Conclusions: Shorter DTE time was associated with better survival at 7 and 28 days, as well as other clinical outcomes, in patients with severe pelvic fracture who underwent TAE. Efforts to minimize DTE time are recommended to improve the clinical outcomes in patients with pelvic fracture treated with TAE.

\section{Background}

The incidence of pelvic fracture in blunt trauma is as high as $10 \%$, with mortality ranging from $21 \%$ to $50 \%$ primarily owing to hemorrhagic shock $[1,2]$. Hemorrhage from pelvic vessels is a dreaded and potentially lethal condition of pelvic fractures [3, 4]. Pelvic transarterial embolization (TAE) is the most effective intervention for management of arterial hemorrhage associated with pelvic fracture [5-7]. TAE has come of age and has an important role in the treatment of patients with pelvic fracture, supported by the highest level of evidence [7-9]; for instance, pelvic angiography with embolization seems to be $85-97 \%$ effective for controlling bleeding $[6,8]$.

Delayed hemorrhage control may increase a patient's mortality risk with time; early angiography with embolization is associated with improved patient outcomes in patients with pelvic fracture [10-13]. However, many previous studies have shown that it is difficult to achieve this goal $[14,15]$.

The current study evaluated the impact of delays in performing pelvic TAE on patients' survival. We hypothesized that a larger door-to-embolization (DTE) time would be significantly associated with increased mortality in patients with severe pelvic fracture.

\section{Methods}

\section{Study setting}

In the Pusan National University Hospital Regional Trauma Center, there are more than 900-1,000 severe traumarelated admissions annually (Injury Severity Score [ISS] $\geq 16$ ), of which 200-250 patients present with pelvic fracture. At our institution, three interventional radiologists and the equipment required for TAE are available $24 \mathrm{~h}$ a day, 7 days a 
week [16]. Thus, the time from arrival to angiography can be less than $2 \mathrm{~h}$. Patients with pelvic fractures without extrapelvic injuries requiring emergency treatment are treated according to the pelvic fracture management algorithm (Figure 1). Indication for TAE is intrapelvic contrast extravasation or hematoma in a computed tomography scan or a transient responder with hemodynamic instability $(\mathrm{HI})$ associated with pelvic fractures. If needed, TAE is also conducted after pelvic packing or any damage control operation or procedures (Figure 1).

\section{Study population}

We retrospectively reviewed data from the medical records and included a total of 1,017 patients with pelvic fracture admitted to the trauma resuscitation unit at our Trauma Center between November 1, 2015 and December 31, 2019. Pelvic injuries almost always accompany injuries to other organ systems. Considering only isolated pelvic injuries would not be realistic; thus, polytraumatic patients with pelvic bone fracture were included in this study. We excluded patients declared dead-on-arrival or discharged or transferred from a trauma resuscitation unit within $24 \mathrm{~h}$ or patients who did not undergo TAE ( $n=702)$ or with unclear medical records $(n=7)$. Patients who underwent angiography more than $12 \mathrm{~h}$ after admission were excluded $(\mathrm{n}=4)$, as they likely had delayed presentation of the indications for TAE or had prolonged periods of time with operative treatment of multiple injuries. We further excluded patients with an Abbreviated Injury Scale (AIS) score for pelvic ring fracture $\leq 2(n=100)$. The final study population included 204 TAE patients (Figure 2).

Available data included age, sex, mechanism of injury, vital sign on arrival, transfusion with packed red blood cells (pRBCs) within $4 \mathrm{~h}$ and $24 \mathrm{~h}$ of arrival, AIS, ISS, Glasgow Coma Scale score (GCS), Revised Trauma Score (RTS), shock index, Trauma-related ISS (TRISS), massive transfusion within initial $24 \mathrm{~h}$ of arrival, hospital length of stay, intensive care unit (ICU) stay, and survival status at 7 days, 28 days, and discharge. Massive transfusion was defined as the replacement by transfusion of 10 units of red blood cells in $24 \mathrm{~h}$.

\section{Definitions and outcome measures}

We defined DTE time as the time from the arrival at hospital to the first application of embolic agents such as polyvinyl alcohol, Gelfoam, coils, and so forth to pelvic arteries. We defined door-to-angiography (DTA) time as that from the arrival at the hospital to the beginning of angiography, injury-to-embolization time as that from the onset of injury to the first application of embolic agents, and injury-to-door time as that from the injury onset to the arrival at hospital (Figure 3). Severe pelvic fracture was defined as a pelvic fracture with an AIS for pelvic ring fracture $\geq 3$. The shock index was defined as heart rate (beat/min)/systolic blood pressure (SBP; mmHg). HI was defined as shock index $\geq 0.9$. Daytime was defined as 8:30 a.m. through 5:30 p.m., and the weekend was defined as 5:31 p.m. Friday through 08:29 a.m. Monday.

The primary outcomes were mortality at day 7 and day 28. Secondary outcomes included overall mortality (in-hospital mortality), pRBC transfusion amounts during the initial $24 \mathrm{~h}$, ICU-free days to day 28, and hospital-free days to day 90. ICU-free days to day 28 were calculated as 28 minus the number of days or part-days in the ICU. All patients who died before the day 28 follow-up were counted as having zero ICU-free days, on the basis that they should be counted as having the worst possible outcome. Hospital-free days to day 90 are a composite of in-hospital death and hospital length of stay, defined as the number of days alive and out of the hospital between the index visit to the trauma resuscitation unit and 90 days later. Patients who died during the index hospitalization and those hospitalized for more than 90 days were classified as having zero hospital-free days. For patients discharged alive before day 90 , the number of hospital-free days was calculated as 90 minus the length of stay. 
We divided the patients into two groups according to their DTE time ( $\leq 150 \mathrm{~min}$ vs. $>150 \mathrm{~min}$ ) to assess the effects of that on clinical outcomes. We arbitrarily set the cut-off point (150 min) at the median of the DTE time.

\section{Statistical analyses}

We present continuous variables as median and interquartile ranges and categorical variables as numbers and percentages. We compared categorical variables using the chi-square test when appropriate; otherwise, we used Fisher's exact test. We compared continuous variables with a Wilcoxon rank-sum test on the basis of the distribution. Multivariate binomial logistic regression analyses were performed in a stepwise fashion, evaluating the effects on mortality of age, SBP, lactic acid, base excess, ISS, GCS, RTS, pRBC transfusion amounts during the initial $24 \mathrm{~h}, \mathrm{DTE}$ time, and injury-to-embolization time. In addition to comparing survival at 7 days and 28 days between DTE time and mortality, Kaplan-Meier plots of survival curves up to 28 days for each group were drawn and their differences were assessed using the log-rank test. We used a multivariate Cox proportional-hazards model to estimate the hazard ratio of DTE time for mortality at day 28 by adjusting for compounding factors. We performed multivariate linear regression analyses to estimate the impact of DTE time on ICU-free days to day 28 , hospital-free days to day 90 , and $24 \mathrm{~h}$ pRBC transfusion requirement. A value of $p<0.05$ was declared to be statistically significant. The Statistical Package for the Social Sciences (Version 20.0, SPSS, Inc., Chicago, IL, USA) and STATA software (Version 14.2, Stata Corp., College Station, TX, USA) were used to analyze the data.

\section{Results}

\section{Demographics of patients with severe pelvic fracture undergoing TAE}

The median DTA time was 107 min (interquartile range [IQR], 79-134). DTA time was not significantly different between daytime and nighttime (98 min [IQR, 74-133] vs. $113 \mathrm{~min}$ [IQR, 86-137], $p=0.057$ ) or between weekday and weekend or holiday (105 min [IQR, 77-133] vs. 113 min [IQR, 86-141], $p=0.167$ ). The median DTE time was 150 min (interquartile range [IQR], 123-186). In addition, the median injury-to-embolization and injury-to-door times were 313 min (IQR, 239-430) and $106 \mathrm{~min}$ (IQR, 40-205), respectively. The median age was 57 years (IQR, 40-70), and 45.1\% were female. The median ISS was 34 (IQR, 25-41) and 70.1\% had HI. The median GCS, RTS, and TRISS were 15 (IQR, 11-15), 7.55 (IQR, 6.38-7.84), and 0.83 (IQR, 0.62-0.94), respectively. Most patients had associated severe injuries (AIS $\geq 3$ ), with head and neck (27.0\%), thoracic (53.4\%), and abdominal (27.3\%) injuries occurring most commonly. The median ICU-free days to day 28 and hospital-free days to day 90 were 21 days (IQR, 0-26) and 46 days (IQR, 063), respectively. The median $24 \mathrm{~h}$ transfusion requirements were five packs (IQR, 2-12) of pRBCs. In addition, the mortality rates at 7 days, at 28 days, and overall were $8.3 \%, 13.7 \%$, and $15.7 \%$, respectively. Interestingly, all deaths due to hemorrhage ( $n=8$ ) occurred within 7 days. The causes of death of the 4 patients who died after 28 days were sepsis or organ failure (3 patients) and traumatic brain injury (1 patient). The demographics of the patients with severe pelvic fracture undergoing TAE are shown in Table 1. 
Table 1

Characteristics of patients treated with transarterial embolization $(\mathrm{n}=$ 204)

\begin{tabular}{|c|c|}
\hline Characteristics & Variable \\
\hline Injury-to-door time, median (IQR), min & $106(40-205)$ \\
\hline Door-to-angiography time, median (IQR), min & $107(79-134)$ \\
\hline Door-to-embolization time, median (IQR), min & $150(123-186)$ \\
\hline Injury-to-embolization time, median (IQR), min & $313(239-430)$ \\
\hline \multicolumn{2}{|l|}{ Origin of admission, $\mathrm{n}(\%)$} \\
\hline Scene & $99(48.5)$ \\
\hline Transfer & $105(51.5)$ \\
\hline \multicolumn{2}{|l|}{ Time of admission } \\
\hline Weekday or day, n (\%) & $63(30.1)$ \\
\hline Weekend or night or holiday, n (\%) & $141(69.1)$ \\
\hline Age, median (IQR), years & $57(40-70)$ \\
\hline Female, n (\%) & $92(45.1)$ \\
\hline \multicolumn{2}{|l|}{ Injury mechanism, n (\%) } \\
\hline Car TA & $14(6.9)$ \\
\hline Motorcycle TA & $20(9.8)$ \\
\hline Pedestrian TA & $75(36.8)$ \\
\hline Fall & $70(34.3)$ \\
\hline Entrapment & $13(6.4)$ \\
\hline Others & $12(5.9)$ \\
\hline \multicolumn{2}{|l|}{ Physiology at admission } \\
\hline Systolic blood pressure, median (IQR), mmHg & $90(70-100)$ \\
\hline Heart rate, median (IQR), beats/min & $94(80-114)$ \\
\hline Shock index, median (IQR) & $1.1(0.8-1.4)$ \\
\hline Hemodynamic instability, n (\%) & $143(70.1)$ \\
\hline Lactic acid, median (IQR), mmol/L & $3.9(2.5-6.3)$ \\
\hline Base excess, median (IQR) & $-4.1(-7.6--0.9)$ \\
\hline ISS, median (IQR) & $34(25-41)$ \\
\hline GCS, median (IQR) & $15(11-15)$ \\
\hline RTS, median (IQR) & $7.55(6.38-7.84)$ \\
\hline TRISS score, median (IQR) & $0.83(0.62-0.94)$ \\
\hline
\end{tabular}




\begin{tabular}{|c|c|}
\hline \multicolumn{2}{|l|}{ Pelvic ring fracture AIS, n (\%) } \\
\hline 3 & $26(12.8)$ \\
\hline 4 & $110(53.9)$ \\
\hline 5 & $68(33.3)$ \\
\hline Head \& neck AIS $\geq 3, \mathrm{n}(\%)$ & $55(27.0)$ \\
\hline Chest AIS $\geq 3, \mathrm{n}(\%)$ & $109(53.4)$ \\
\hline Abdomen AIS $\geq 3, \mathrm{n}(\%)$ & $76(37.3)$ \\
\hline Any surgery, n (\%) & $184(90.2)$ \\
\hline Any surgery within 24 h, n (\%) & $77(37.7)$ \\
\hline Pelvis surgery within $24 \mathrm{~h}, \mathrm{n}(\%)$ & $24(11.8)$ \\
\hline Pelvic stabilization (external fixation), $\mathrm{n}(\%)^{*}$ & $7(29.2)$ \\
\hline Preperitoneal packing, n (\%) & $6(2.9)$ \\
\hline \multicolumn{2}{|l|}{ Outcome } \\
\hline 28-day free ICU stay, median (IQR), days & $21(0-26)$ \\
\hline 90-day free hospital stay, median (IQR), days & $46(0-63)$ \\
\hline \multicolumn{2}{|l|}{ pRBC transfusion } \\
\hline$\leq 4 \mathrm{~h}$ pRBC transfusion, median (IQR), packs & $3(1-6)$ \\
\hline 4-24 h pRBC transfusion, median (IQR), packs & $2(0-5)$ \\
\hline $24 \mathrm{~h}$ pRBC transfusion, median (IQR), packs & $5(2-12)$ \\
\hline MT within $4 \mathrm{~h}$ ( $\geq 10$ packs pRBC), $\mathrm{n}(\%)$ & $16(7.8)$ \\
\hline MT between 4-24 h ( $\geq 10$ packs pRBC), n (\%) & $25(12.8)$ \\
\hline MT within $24 \mathrm{~h}$ ( $\geq 10$ packs pRBC), n (\%) & $61(29.9)$ \\
\hline Mortality at 7 days, n (\%) & $17(8.3)$ \\
\hline Hemorrhage, $n(\%)^{\dagger}$ & $8(47.1)$ \\
\hline Sepsis or organ failure, $\mathrm{n}(\%)^{\dagger}$ & $4(23.5)$ \\
\hline Traumatic brain injury, $\mathrm{n}(\%)^{\dagger}$ & $4(23.5)$ \\
\hline Others, $\mathrm{n}(\%)^{\dagger}$ & $1(5.9)$ \\
\hline Mortality at 28 days, n (\%) & $28(13.7)$ \\
\hline Hemorrhage, $n(\%)^{\dagger}$ & $8(28.6)$ \\
\hline Sepsis or organ failure, $\mathrm{n}(\%)^{\dagger}$ & $10(35.7)$ \\
\hline Traumatic brain injury, $\mathrm{n}(\%)^{\dagger}$ & $8(28.6)$ \\
\hline Others, n (\%) ${ }^{\dagger}$ & $2(7.1)$ \\
\hline
\end{tabular}




\begin{tabular}{|c|c|}
\hline Overall mortality, n (\%) & $32(15.7)$ \\
\hline Hemorrhage, $\mathrm{n}(\%)^{\dagger}$ & $8(25)$ \\
\hline Sepsis or organ failure, $\mathrm{n}(\%)^{\dagger}$ & $13(40.6)$ \\
\hline Traumatic brain injury, $\mathrm{n}(\%)^{\dagger}$ & $9(28.1)$ \\
\hline Others, $\mathrm{n}(\%)^{\dagger}$ & $2(6.3)$ \\
\hline
\end{tabular}

${ }^{*}$ Attributable percentage of total pelvis surgery within $24 \mathrm{~h} .{ }^{\dagger}$ Attributable percentage of total mortality

\section{Risk factors for mortality at 7 days (early mortality) (Table 2)}

The median DTE, injury-to-door, and injury-to-embolization times were not significantly different between the mortality and non-mortality groups whereas the median DTA time was significantly larger in the mortality group. In addition, patients who died were significantly older and had lower SBP upon arrival, higher incidence of HI, higher levels of lactic acid, lower base excess, higher ISS, lower GCS, lower RTS score, and greater requirement for $24 \mathrm{~h}$ pRBC transfusion within $24 \mathrm{~h}$. The two groups did not significantly differ in sex, AIS for head and neck, AIS for chest, AIS for abdomen, AIS for pelvic ring fracture, or other standard resuscitation or operation. 
Table 2

Clinical features according to early mortality (mortality within 7 post-trauma days; $n=204$ )

\begin{tabular}{|c|c|c|c|c|c|}
\hline Variable & $\begin{array}{l}\text { Non-mortality at } 7 \text { days } \\
\text { group }(n=187)\end{array}$ & $\begin{array}{l}\text { Mortality at } 7 \\
\text { days group } \\
(n=17)\end{array}$ & $p$-value & $\begin{array}{l}\text { Adjusted odds } \\
\text { ratio* }^{*}(95 \% \mathrm{Cl})\end{array}$ & $p$-value \\
\hline $\begin{array}{l}\text { Door-to-embolization time, } \\
\text { median (IQR), h }\end{array}$ & $2.5(2.0-3.1)$ & $2.7(2.5-7.2)$ & 0.153 & $\begin{array}{l}1.62(1.14- \\
2.30)\end{array}$ & 0.007 \\
\hline $\begin{array}{l}\text { Door-to-angiography time, } \\
\text { median (IQR), h }\end{array}$ & $1.8(1.2-2.2)$ & $2.1(1.8-5.9)$ & 0.037 & & \\
\hline $\begin{array}{l}\text { Injury-to-door time, median } \\
(\text { IQR), h }\end{array}$ & $1.8(0.6-3.5)$ & $1.6(0.7-3.0)$ & 0.494 & & \\
\hline $\begin{array}{l}\text { Injury-to-embolization time, } \\
\text { median (IQR), h }\end{array}$ & $5.2(4.0-7.2)$ & $5.6(4.0-9.7)$ & 0.369 & & \\
\hline Origin of admission, $\mathrm{n}(\%)$ & & & 0.526 & & \\
\hline Scene & $92(49.2)$ & $7(41.2)$ & & & \\
\hline Transfer & $95(50.8)$ & $10(58.8)$ & & & \\
\hline Age, median (IQR), years & $56(39-69)$ & $70(53-75)$ & 0.046 & $\begin{array}{l}1.06(1.01- \\
1.12)\end{array}$ & 0.022 \\
\hline Female, n (\%) & $85(45.5)$ & $7(41.2)$ & 0.734 & & \\
\hline \multicolumn{6}{|l|}{ Physiology at admission } \\
\hline $\begin{array}{l}\text { Systolic blood pressure, } \\
\text { median (IQR), mmHg }\end{array}$ & $90(70-100)$ & $60(50-80)$ & $<0.001$ & & \\
\hline $\begin{array}{l}\text { Heart rate, median (IQR), } \\
\text { beats/min }\end{array}$ & $94(80-114)$ & $88(70-116)$ & 0.488 & & \\
\hline $\begin{array}{l}\text { Hemodynamic instability, } \mathrm{n} \\
(\%)\end{array}$ & $127(67.9)$ & $16(94.1)$ & 0.024 & & \\
\hline $\begin{array}{l}\text { Lactic acid, median (IQR), } \\
\text { mmol/L }\end{array}$ & $3.7(2.3-5.6)$ & $7.0(4.3-9.0)$ & $<0.001$ & & \\
\hline Base excess, median (IQR) & $-3.9(-7.2--0.9)$ & $\begin{array}{l}-8.3(-11.3- \\
-4.3)\end{array}$ & 0.018 & & \\
\hline ISS, median (IQR) & $30(25-41)$ & $41(36-50)$ & $<0.001$ & & \\
\hline GCS, median (IQR) & $15(13-15)$ & $9(6-14)$ & $<0.001$ & & \\
\hline RTS, median (IQR) & $7.84(6.37-7.84)$ & $\begin{array}{l}6.37(5.23- \\
6.61)\end{array}$ & $<0.001$ & $\begin{array}{l}0.64(0.42- \\
0.97)\end{array}$ & 0.037 \\
\hline $\begin{array}{l}\text { AIS for pelvic ring fracture, } n \\
(\%)\end{array}$ & & & 0.824 & & \\
\hline 3 & $24(12.8)$ & $2(11.7)$ & & & \\
\hline 4 & $102(54.6)$ & $8(47.1)$ & & & \\
\hline 5 & $61(32.6)$ & $7(41.2)$ & & & \\
\hline Head \& neck AIS $\geq 3, \mathrm{n}(\%)$ & $48(25.7)$ & 7 (41.2) & 0.251 & & \\
\hline
\end{tabular}




\begin{tabular}{|c|c|c|c|c|c|}
\hline Chest AIS $\geq 3, n(\%)$ & $97(51.9)$ & $12(70.6)$ & 0.139 & & \\
\hline Abdomen AlS $\geq 3, n$ (\%) & 66 (35.3) & $10(58.8)$ & 0.055 & & \\
\hline \multicolumn{6}{|l|}{ Operation, n (\%) } \\
\hline Any operation within $24 \mathrm{~h}$ & $68(36.6)$ & $9(52.9)$ & 0.183 & & \\
\hline Pelvis surgery within $24 \mathrm{~h}$ & $24(12.9)$ & 0 & 0.231 & & \\
\hline PPP & $5(2.7)$ & $1(5.9)$ & 0.411 & & \\
\hline $\begin{array}{l}\text { pRBC transfusion within } 24 \mathrm{~h} \text {, } \\
\text { median (IQR), packs }\end{array}$ & $4(2-11)$ & $14(9-18)$ & $<0.001$ & $\begin{array}{l}1.05(1.00- \\
1.10)\end{array}$ & 0.047 \\
\hline
\end{tabular}

*adjusted odds ratios for age, systolic blood pressure, lactic acid, base excess, ISS, GCS, RTS, abdomen AIS $\geq 3$, and pRBC transfusion in the initial $24 \mathrm{~h}$

Multivariate logistic regression analyses were performed to evaluate the independent risk factors for mortality at 7 days. After adjusting for nine variables (age, SBP upon arrival, HI, lactic acid, base excess, ISS, GCS, RTS, and pRBC transfusion requirement in the initial $24 \mathrm{~h}$ ), DTE time served as an independent risk factor for mortality at 7 days. An increase of $1 \mathrm{~h}$ in DTE time resulted in a 1.62-fold increase in mortality at 7 days.

\section{Risk factors for mortality at 28 days (Table 3 )}

In univariate analyses, factors associated with mortality at 28 days of patients with severe pelvic fracture were DTE time, age, SBP at admission, HI, lactic acid, ISS, GCS, RTS, AIS for head and neck $\geq 3$, and pRBC transfusion amounts in the initial $24 \mathrm{~h}$. After adjusting for the nine variables, DTE time was an independent risk factor for mortality at 28 days. An increase of $1 \mathrm{~h}$ in DTE time resulted in a 1.48-fold increase in mortality at 28 days. 
Table 3

Univariate and multivariate logistic regression analyses for mortality at 28 days $(n=204)$

\begin{tabular}{|c|c|c|c|c|}
\hline Variable & $\begin{array}{l}\text { Crude odds ratio }(95 \% \\
\mathrm{Cl})\end{array}$ & $p$-value & $\begin{array}{l}\text { Adjusted odds } \\
\text { ratio* }^{*} \\
(95 \% \mathrm{Cl})\end{array}$ & $p$-value \\
\hline Door-to-embolization time, median (IQR), h & $1.31(1.07-1.60)$ & 0.009 & $1.48(1.05-2.07)$ & 0.023 \\
\hline Door-to-angiography time, median (IQR), $\mathrm{h}$ & $1.32(1.07-1.63)$ & 0.007 & & \\
\hline Injury-to-embolization time, median (IQR), h & $1.02(0.93-1.12)$ & 0.733 & & \\
\hline Injury-to-door time, median (IQR), h & $0.94(0.80-1.10)$ & 0.394 & & \\
\hline Age, median (IQR), years & $1,02(1.00-1.05)$ & 0.032 & $1.06(1.01-1.10)$ & 0.009 \\
\hline Female, n (\%) & $0.90(0.40-2.01)$ & 0.798 & & \\
\hline \multicolumn{5}{|l|}{ Physiology at admission } \\
\hline Systolic blood pressure, median (IQR), mmHg & $0.98(0.97-0.99)$ & 0.001 & & \\
\hline Heart rate, median (IQR), beats/min & $1.00(0.98-1.01)$ & 0.907 & & \\
\hline Hemodynamic instability, n (\%) & $4.09(1.19-14.13)$ & 0.025 & & \\
\hline Lactic acid, median (IQR), mmol/L & $1.09(1.00-1.18)$ & 0.047 & & \\
\hline Base excess, median (IQR) & $0.95(0.89-1.00)$ & 0.072 & & \\
\hline ISS, median (IQR) & $1.09(1.05-1.14)$ & $<0.001$ & $1.08(1.01-1.15)$ & 0.017 \\
\hline GCS, median (IQR) & $0.81(0.74-0.89)$ & $<0.001$ & & \\
\hline RTS, median (IQR) & $0.65(0.49-0.85)$ & 0.002 & $0.67(0.47-0.95)$ & 0.026 \\
\hline \multicolumn{5}{|l|}{ AIS for pelvic ring fracture, $\mathrm{n}(\%)$} \\
\hline 3 & reference & & & \\
\hline 4 & $1.47(0.31-7.00)$ & 0.629 & & \\
\hline 5 & $3.11(0.65-14.77)$ & 0.153 & & \\
\hline Head \& neck AIS $\geq 3, n(\%)$ & $2.32(1.02-5.29)$ & 0.045 & & \\
\hline Chest AIS $\geq 3, \mathrm{n}(\%)$ & $1.68(0.73-3.85)$ & 0.219 & & \\
\hline Abdomen AIS $\geq 3, \mathrm{n}(\%)$ & $1.84(0.82-4.10)$ & 0.137 & & \\
\hline $\begin{array}{l}\text { pRBC transfusion within } 24 \mathrm{~h} \text {, median (IQR), } \\
\text { packs }\end{array}$ & $1.11(1.06-1.17)$ & $<0.001$ & $1.10(1.03-1.19)$ & 0.005 \\
\hline
\end{tabular}

*adjusted odds ratio for age, systolic blood pressure, lactic acid, base excess, ISS, GCS, RTS, head and neck AIS $\geq 3$, and $\mathrm{pRBC}$ transfusion in the initial $24 \mathrm{~h}$

Multivariate Cox proportional hazards regression analyses were performed to evaluate the independent risk factors for mortality at 28 days. After adjusting for age, SBP upon arrival, lactic acid levels, base excess, ISS, GCS, RTS, and pRBC transfusion amounts during the initial $24 \mathrm{~h}$, the adjusted hazard ratio of DTE time was 1.28. This means that an increase of $1 \mathrm{~h}$ in DTE time resulted in a 1.28-fold increase in mortality at 28 days. 


\section{Secondary outcomes of patients according to DTE time}

Multivariate linear regression analyses were performed to evaluate the effects of DTE time on pRBC transfusion requirement in the initial $24 \mathrm{~h}$, ICU-free days to day 28 , and hospital-free days to day 90 . DTE time was an independent indicator of $24 \mathrm{~h}$ pRBC transfusion requirement and ICU-free days to day 28 ( $p=0.045$ and 0.026 , respectively; Table 4). Figure 4 shows the positive relationship between DTE time and pRBC transfusion amounts in the initial $24 \mathrm{~h}$ and the negative relationship between DTE time and ICU-free days to day 28. However, no significant difference in hospitalfree days to day 90 was found. Similarly, overall mortality was not significantly different in multivariate logistic regression analyses (adjusted $\mathrm{OR}=1.35,95 \% \mathrm{Cl}=0.98-1.85, p=0.067$ ).

Table 4

Secondary outcomes according to door-to-embolization time $(\mathrm{n}=204)$

\begin{tabular}{|c|c|c|c|c|c|c|c|c|}
\hline & $\begin{array}{l}\text { Unstandardized } \\
\text { coefficient }^{\dagger}\end{array}$ & $\begin{array}{l}\text { Standard } \\
\text { error }\end{array}$ & $\begin{array}{l}\text { Standardized } \\
\text { coefficients } \\
\text { beta }^{+}\end{array}$ & $p$-value & $\begin{array}{l}\text { Crude } \\
\text { OR } \\
(95 \% \\
\text { Cl) }\end{array}$ & $p$-value & $\begin{array}{l}\text { Adjusted } \\
\text { OR } \\
(95 \% \mathrm{Cl})\end{array}$ & $p$-value \\
\hline $\begin{array}{l}\mathrm{pRBC} \\
\text { transfusion } \\
\text { requirement } \\
\text { in the initial } \\
24 \mathrm{~h}^{*}\end{array}$ & 1.032 & 0.512 & 0.133 & 0.045 & & & & \\
\hline $\begin{array}{l}\text { ICU-free } \\
\text { days to day } \\
28^{*}\end{array}$ & -0.922 & 0.410 & -0.134 & 0.026 & & & & \\
\hline $\begin{array}{l}\text { Hospital- } \\
\text { free days to } \\
\text { day } 90^{*}\end{array}$ & -1.334 & 1.130 & -0.075 & 0.239 & & & & \\
\hline $\begin{array}{l}\text { Overall } \\
\text { mortality }\end{array}$ & & & & & $\begin{array}{l}1.26 \\
(1.04- \\
1.53)\end{array}$ & 0.021 & $\begin{array}{l}1.35 \\
(0.98- \\
1.86)^{\ddagger}\end{array}$ & 0.067 \\
\hline
\end{tabular}

*door-to-embolization time per $1 \mathrm{~h}$ increase. tadjusted coefficient for ISS, age, hemodynamic instability (shock index $\geq$ 0.9 ), and AIS for pelvic ring fracture. ładjusted odds ratio for age, systolic blood pressure, hemodynamic instability, lactic acid, base excess, ISS, GCS, RTS, and pRBC transfusion in the initial $24 \mathrm{~h}$

\section{Subgroup analyses: clinical outcomes according to DTE time ( $\leq 150 \mathrm{~min}$ vs. $>150 \mathrm{~min})$}

We divided the patients into two groups according to their DTE time to assess the effects of this factor on clinical outcomes. We set the cut-off point as 150 min, which was the median DTE time. Table 5 shows the clinical outcomes for these groups: patients with a DTE time of more than 150 min showed higher mortality at 7 days, at 28 days, and overall, and fewer ICU-free days to day 28 than patients with a DTE time less than or equal 150 min. However, no significant differences in pRBC transfusion requirement or hospital-free days to day 90 were found between the groups. 
Table 5

Clinical outcomes according to door-to-embolization time ( $\leq 150$ min vs. $>150$ min; $n=204$ )

\begin{tabular}{|c|c|c|c|c|c|c|c|c|}
\hline & $\begin{array}{l}\text { Unstandardized } \\
\text { coefficient }^{\dagger}\end{array}$ & $\begin{array}{l}\text { Standard } \\
\text { error }\end{array}$ & $\begin{array}{l}\text { Standardized } \\
\text { coefficients } \\
\text { beta }^{+}\end{array}$ & $p$-value & $\begin{array}{l}\text { Crude } \\
\text { OR } \\
(95 \% \\
\text { Cl) }\end{array}$ & $p$-value & $\begin{array}{l}\text { Adjusted } \\
\mathrm{OR}^{\ddagger} \\
(95 \% \mathrm{Cl})\end{array}$ & $p$-value \\
\hline $\begin{array}{l}\text { Mortality at } \\
7 \text { days }^{*}\end{array}$ & & & & & $\begin{array}{l}2.53 \\
(0.96- \\
7.47)\end{array}$ & 0.092 & $\begin{array}{l}4.24 \\
(1.00- \\
17.93)\end{array}$ & 0.049 \\
\hline $\begin{array}{l}\text { Mortality at } \\
28 \text { days }^{*}\end{array}$ & & & & & $\begin{array}{l}2.31 \\
(0.99- \\
5.39)\end{array}$ & 0.052 & $\begin{array}{l}3.35 \\
(1.01- \\
11.13)\end{array}$ & 0.048 \\
\hline $\begin{array}{l}\text { Overall } \\
\text { mortality* }\end{array}$ & & & & & $\begin{array}{l}2.10 \\
(0.95- \\
4.61)\end{array}$ & 0.066 & $\begin{array}{l}3.28 \\
(1.05- \\
10.23)\end{array}$ & 0.040 \\
\hline $\begin{array}{l}\text { pRBC } \\
\text { transfusion } \\
\text { requirement } \\
\text { in the initial } \\
24 \mathrm{~h}^{\star}\end{array}$ & -0.148 & 1.622 & -0.006 & 0.927 & & & & \\
\hline $\begin{array}{l}\text { 28-day ICU } \\
\text { free days* }\end{array}$ & -2.594 & 1.287 & -0.119 & 0.045 & & & & \\
\hline $\begin{array}{l}\text { 90-day } \\
\text { hospital } \\
\text { free days* }\end{array}$ & -0.971 & 3.555 & -0.017 & 0.785 & & & & \\
\hline
\end{tabular}

*door-to-embolization time $\leq 150 \mathrm{~min}$ vs. >150 min; reference group: $\leq 150 \mathrm{~min}$. tadjusted coefficient for ISS, age, hemodynamic instability, and pelvis AIS. ładjusted odds ratio for age, systolic blood pressure, hemodynamic instability, lactic acid, base excess, ISS, GCS, RTS, and pRBC transfusion in the initial $24 \mathrm{~h}$ 
Table 6

Summary of reported series about the impact of door-to-embolization time on the mortality of patients with pelvic fracture undergoing transarterial embolization (TAE)

\begin{tabular}{|c|c|c|c|c|}
\hline $\begin{array}{l}\text { Study } \\
\text { citation } \\
\text { (year) }\end{array}$ & $\begin{array}{l}\text { No. } \\
\text { of } \\
\text { TAE } \\
\text { cases }\end{array}$ & $\begin{array}{l}\text { Outcome } \\
\text { variable }\end{array}$ & Time (min) & Impact on mortality \\
\hline $\begin{array}{l}\text { Agolini et al. } \\
\text { [11] (1997) }\end{array}$ & 15 & $\begin{array}{l}\text { Time from } \\
\text { arrival to } \\
\text { angiography } \\
\text { suite }\end{array}$ & $\begin{array}{l}190 \min \\
(I Q R, 50- \\
1440)\end{array}$ & $\begin{array}{l}\text { Patients who were in the angiography suite within } 3 \mathrm{~h} \\
\text { of arrival had a significantly greater survival rate ( } 14 \\
\text { vs. } 75 \% \text { ) }\end{array}$ \\
\hline $\begin{array}{l}\text { Balogh et al. } \\
\text { [12] (2005) }\end{array}$ & 31 & DTA time & $\begin{array}{l}<90 \text { min } \\
\text { after } \\
\text { admission }\end{array}$ & $\begin{array}{l}\text { Institutional protocol improving time to angiography to } \\
\text { less than } 90 \text { min decreased mortality from } 35 \text { to } 7 \% \text { ( } \\
<0.05 \text { ) }\end{array}$ \\
\hline $\begin{array}{l}\text { Schwartz et } \\
\text { al.[19] (2014) }\end{array}$ & 88 & $\begin{array}{l}\text { Time from } \\
\text { admission to } \\
\text { angiography } \\
\text { suite }\end{array}$ & $\begin{array}{l}\text { Day: } 193 \\
\text { min (IQR, } \\
\text { 137-275), } \\
\text { after-hours: } \\
301 \text { min } \\
\text { (IQR, } 211- \\
\text { 389) }\end{array}$ & $\begin{array}{l}\text { Delays to angiography in after-hours admission were } \\
\text { associated with higher mortality ( } 32 \text { vs. } 21 \%, p= \\
0.328 \text { ) }\end{array}$ \\
\hline $\begin{array}{l}\text { Tanizaki et } \\
\text { al.[13] (2014) }\end{array}$ & 68 & $\begin{array}{l}\text { Time from } \\
\text { arrival to } \\
\text { angiography } \\
\text { suite }\end{array}$ & $\begin{array}{l}\text { Average of } \\
76 \text { min }(30- \\
145)\end{array}$ & $\begin{array}{l}\text { Patients who were embolized within } 60 \text { min of arrival } \\
\text { had a significantly lower mortality rate ( } 16 \text { vs. } 64 \%, p= \\
0.04)\end{array}$ \\
\hline $\begin{array}{l}\text { Tesoriero et } \\
\text { al.[15] (2017) }\end{array}$ & 212 & DTA time & $\begin{array}{l}280 \min \\
(\text { IQR, 201- } \\
367)\end{array}$ & $\begin{array}{l}\text { Time to angiography was not a significant contributor } \\
\text { to mortality after adjusting for injury severity. }\end{array}$ \\
\hline $\begin{array}{l}\text { Marsushuma } \\
\text { et al. [10] } \\
(2018)\end{array}$ & 181 & DTE time & $\begin{array}{l}\text { [Not } \\
\text { applicable] }\end{array}$ & $\begin{array}{l}\text { A longer time to TAE was significantly associated with } \\
\text { increased in-hospital mortality }(\mathrm{OR}=1.79 \text { for each } \\
\text { hour, } 95 \% \mathrm{Cl}=1.12-2.91, \mathrm{p}=0.018)\end{array}$ \\
\hline $\begin{array}{l}\text { Chou et al. } \\
\text { [14] (2019) }\end{array}$ & 84 & DTE time & $\begin{array}{l}62.0 \pm 33.4 \\
\min \end{array}$ & $\begin{array}{l}\text { There were no significant differences in the time to TAE } \\
\text { between nonsurviving and surviving patients }(76.9 \pm \\
47.9 \text { vs. } 59.0 \pm 29.3 \text { min, } p=0.068)\end{array}$ \\
\hline \multirow[t]{2}{*}{$\begin{array}{l}\text { This study } \\
\text { (2020) }\end{array}$} & 204 & DTE time & $\begin{array}{l}150 \text { min } \\
(\mathrm{IQR}, 123- \\
186)\end{array}$ & $\begin{array}{l}\text { An increase in } 1 \mathrm{~h} \text { in door-to-embolization time resulted } \\
\text { in a } 1.62 \text {-fold increase in mortality at } 7 \text { days }(\mathrm{p}= \\
0.007) \text {. }\end{array}$ \\
\hline & & & & $\begin{array}{l}\text { An increase in } 1 \mathrm{~h} \text { in door-to-embolization time resulted } \\
\text { in a } 1.48 \text {-fold increase in mortality at } 28 \text { days }(p= \\
0.023)\end{array}$ \\
\hline
\end{tabular}

Figure 5 shows the Kaplan-Meier 28-day mortality curves of patients undergoing TAE according to DTE time. The incidence of 28-day mortality was significantly lower in patients with DTE time $\leq 150$ min than in patients with DTE time $>150 \min (p=0.029)$ and a similar result remained even after we divided patients into three groups according to their DTE time ( $\leq 150$ vs. $150-300$ vs. $>300$ min; $p<0.001$; Figure 5).

\section{Conclusion}

Shorter DTE time was associated with better clinical outcomes in patients with severe pelvic fracture who underwent TAE. These findings suggest that shortening DTE time could reduce patients' mortality rate as well as other outcomes, such as blood transfusion requirement and ICU length of stay. Thus, DTE time is an important factor to consider when 
treating patients with suspected pelvic hemorrhage. Efforts to minimize DTE time are recommended to improve the clinical outcomes in patients with pelvic fracture treated with TAE.

\section{Abbreviations}

AIS: Abbreviated Injury Scale score; ATLS: Adult Trauma Life Support; Cl: confidence interval; DTA: door-to-angiography; DTE: door-to-embolization; GCS: Glasgow Coma Scale score; ICU: intensive care unit; IQR: interquartile range; ISS: Injury Severity Score; MT: massive transfusion; OR: odds ratio; PPP: preperitoneal packing; pRBC: packed red blood cells; RTS: Revised Trauma Score; TA: traffic accident; TRISS: Trauma-related Injury Severity Score.

\section{Declaration}

\section{Acknowledgements}

This work was supported by a clinical research grant from Pusan National University Hospital in 2021.

\section{Funding}

No commercial associations with or sources of support from any funding agency.

\section{Availability of data and materials}

Not applicable.

\section{Authors' contributions}

Study conception and design: K-HH, J-CH, and K-JH. Acquisition of the data: K-HH, J-CH, K-H, K-CW, K-GH, L-SB, J-JH and Y-SR. Analysis and interpretation of the data: $\mathrm{K}-\mathrm{HH}$ and $\mathrm{J}-\mathrm{CH}$. Drafting of the manuscript: $\mathrm{K}-\mathrm{HH}$. Critical revision: $\mathrm{L}-$ $\mathrm{CK}, \mathrm{K}-\mathrm{SH}, \mathrm{J}-\mathrm{CH}$ and $\mathrm{K}-\mathrm{JH}$. All authors read and approved the final manuscript.

\section{Ethics approval and consent to participate}

This retrospective study was approved by the Institutional of Review Board of Pusan National University Hospital (H2004-017-090).

\section{Consent for publication}

All authors agree with the publication of this article.

\section{Competing interests}

The authors declare that they have no competing interests.

The English in this document has been checked by at least two professional editors, both native speakers of English. For a certificate, please see:

http://www.textcheck.com/certificate/iNdw4n

\section{References}


1. Costantini TW, Coimbra R, Holcomb JB, Podbielski JM, Catalano R, Blackburn A et al. Current management of hemorrhage from severe pelvic fractures: Results of an American Association for the Surgery of Trauma multiinstitutional trial. J Trauma Acute Care Surg. 2016;80(5):717-23; discussion 23-5.

2. Ghaemmaghami V, Sperry J, Gunst M, Friese R, Starr A, Frankel H et al. Effects of early use of external pelvic compression on transfusion requirements and mortality in pelvic fractures. Am J Surg. 2007;194(6):720-3.

3. Hauschild O, Aghayev E, von Heyden J, Strohm PC, Culemann U, Pohlemann T et al. Angioembolization for pelvic hemorrhage control: Results from the German pelvic injury register. J Trauma Acute Care Surg. 2012;73(3):67984. doi:10.1097/TA.0b013e318253b5ba.

4. White CE, Hsu JR, Holcomb JB. Haemodynamically unstable pelvic fractures. Injury. 2009;40(10):1023-30.

5. Salcedo ES, Brown IE, Corwin MT, Galante JM. Pelvic angioembolization in trauma-Indications and outcomes. Int J Surg. 2016;33(Pt B):231-6. doi:10.1016/j.ijsu.2016.02.057.

6. Vaidya R, Waldron J, Scott A, Nasr K. Angiography and Embolization in the Management of Bleeding Pelvic Fractures. J Am Acad Orthop Surg. 2018;26(4):e68-e76..

7. Coccolini F, Stahel PF, Montori G, Biffl W, Horer TM, Catena F et al. Pelvic trauma: WSES classification and guidelines. World J Emerg Surg. 2017;12:5.

8. Cullinane DC, Schiller HJ, Zielinski MD, Bilaniuk JW, Collier BR, Como J et al. Eastern Association for the Surgery of Trauma practice management guidelines for hemorrhage in pelvic fracture-update and systematic review. J Trauma. 2011;71(6):1850-68.

9. Chu CH, Tennakoon L, Maggio PM, Weiser TG, Spain DA, Staudenmayer KL. Trends in the management of pelvic fractures, 2008-2010. J Surg Res. 2016;202(2):335-40.

10. Matsushima K, Piccinini A, Schellenberg M, Cheng V, Heindel P, Strumwasser A et al. Effect of door-toangioembolization time on mortality in pelvic fracture: Every hour of delay counts. J Trauma Acute Care Surg. 2018;84(5):685-92.

11. Agolini SF, Shah K, Jaffe J, Newcomb J, Rhodes M, Reed JF, 3rd. Arterial embolization is a rapid and effective technique for controlling pelvic fracture hemorrhage. J Trauma. 1997;43(3):395-9.

12. Balogh Z, Caldwell E, Heetveld M, D’Amours S, Schlaphoff G, Harris I et al. Institutional practice guidelines on management of pelvic fracture-related hemodynamic instability: Do they make a difference? J Trauma. 2005;58(4):778-82.

13. Tanizaki S, Maeda S, Matano H, Sera M, Nagai H, Ishida H. Time to pelvic embolization for hemodynamically unstable pelvic fractures may affect the survival for delays up to $60 \mathrm{~min}$. Injury-International Journal of the Care of the Injured. 2014;45(4):738-41.

14. Chou $\mathrm{CH}$, Wu YT, Fu CY, Liao CH, Wang SY, Bajani F et al. Hemostasis as soon as possible? The role of the time to angioembolization in the management of pelvic fracture. World J Emerg Surg. 2019;14. doi:ARTN 28

15. Tesoriero RB, Bruns BR, Narayan M, Dubose J, Guliani SS, Brenner ML et al. Angiographic embolization for hemorrhage following pelvic fracture: Is it "time" for a paradigm shift? J Trauma Acute Care Surg. 2017;82(1):1826.

16. Lee R, Jeon $\mathrm{CH}$, Kim CW, Kwon H, Kim JH, Kim H et al. Clinical Results of Distal Embolization in Grade V Splenic Injury: Four-Year Experience from a Single Regional Trauma Center. J Vasc Interv Radiol. 2020. [Epub ahead of print]

17. Costantini TW, Bosarge PL, Fortlage D, Bansal V, Coimbra R. Arterial embolization for pelvic fractures after blunt trauma: Are we all talk? Am J Surg. 2010;200(6):752-7; discussion 7-8. 
18. Hak DJ, Smith WR, Suzuki T. Management of hemorrhage in life-threatening pelvic fracture. J Am Acad Orthop Surg. 2009;17(7):447-57.

19. Schwartz DA, Medina M, Cotton BA, Rahbar E, Wade CE, Cohen AM et al. Are we delivering two standards of care for pelvic trauma? Availability of angioembolization after hours and on weekends increases time to therapeutic intervention. J Trauma Acute Care. 2014;76(1):134-9.

20. Ito K, Nagao T, Tsunoyama T, Kono K, Tomonaga A, Nakazawa K et al. Hybrid emergency room system improves timeliness of angioembolization for pelvic fracture. J Trauma Acute Care Surg. 2020;88(2):314-9.

21. Gansslen A, Giannoudis P, Pape HC. Hemorrhage in pelvic fracture: Who needs angiography? Curr Opin Crit Care. 2003;9(6):515-23.

22. Evers BM, Cryer HM, Miller FB. Pelvic fracture hemorrhage. Priorities in management. Arch Surg. 1989;124(4):4224.

\section{Figures}

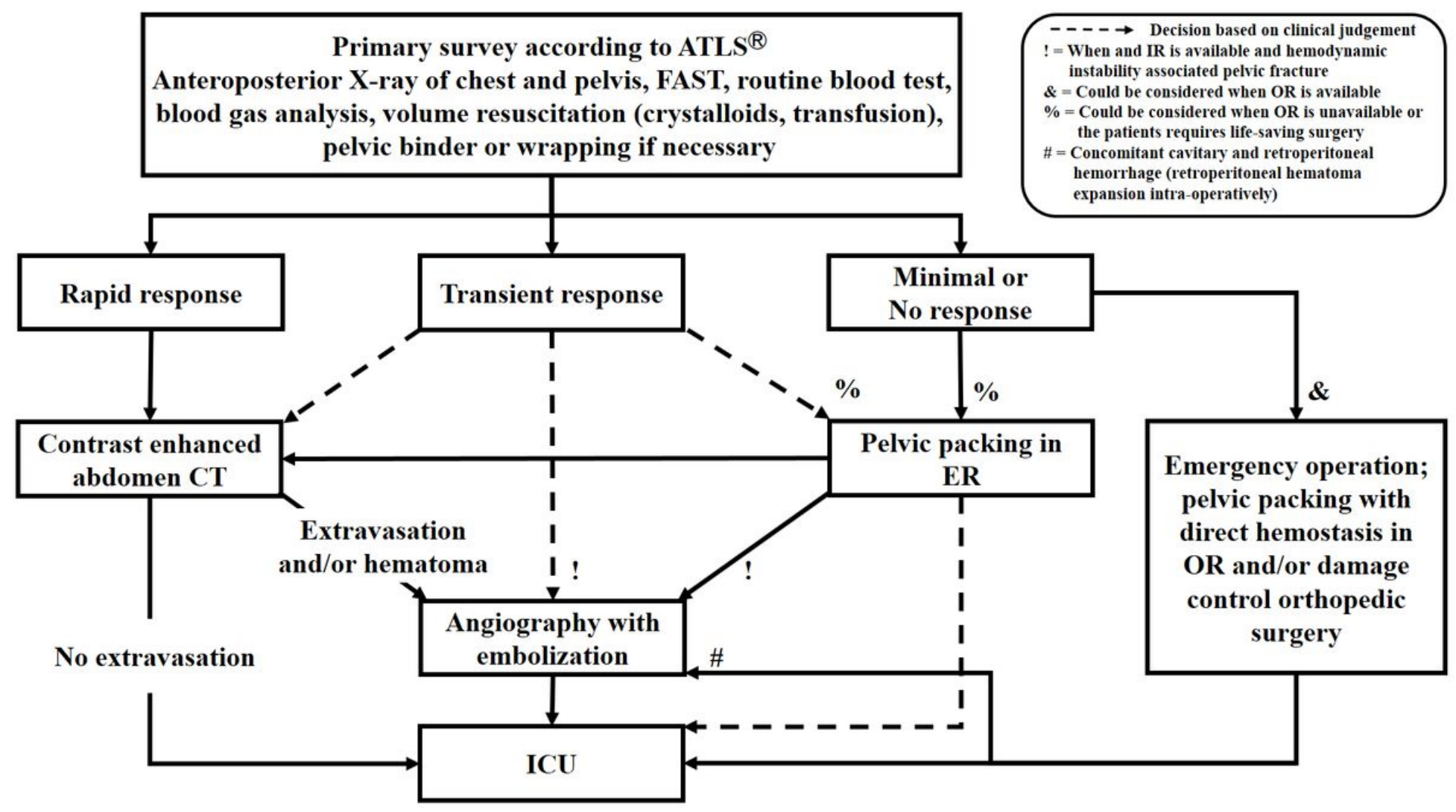

Figure 1

Pelvic fracture management algorithm 
1,017 traumatic pelvic injury patients in the database

15 patients included in analysis

TAE = 315 (31.0\%)

702 patients were excluded due to death on arrival or discharge or transfer from ED, or non-TAE group

7 patients were excluded due to unclear medical records

308 patients included in analysis

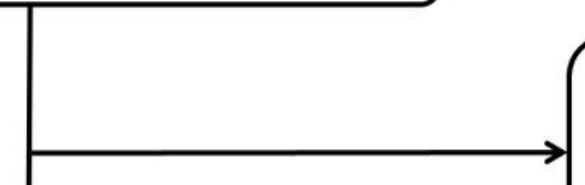

104 patients were excluded due to less than or equal to 2 pelvic bone injury AIS or more than 12 hours from ED arrival to angiography

204 patients included in analysis

Figure 2

Flowchart of the study

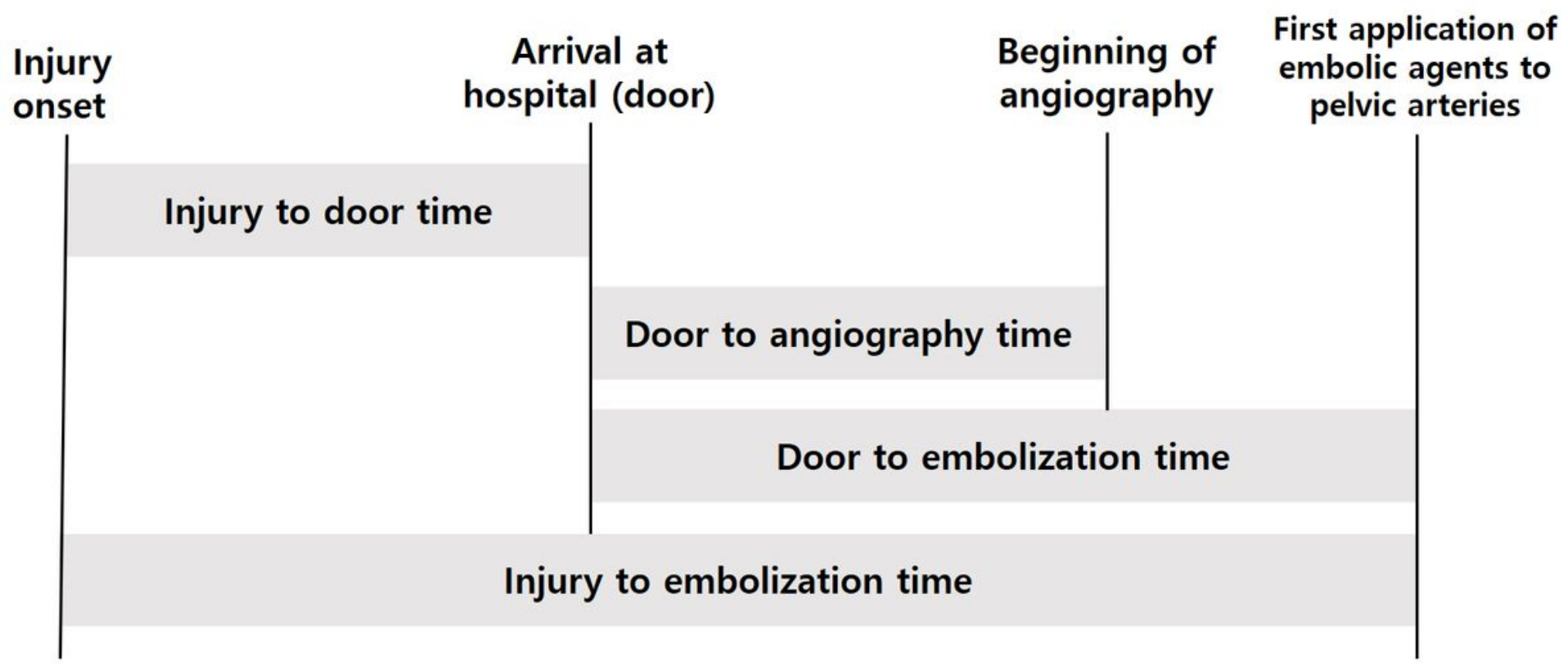

Figure 3 
Scheme for timeframe from injury onset to transarterial embolization in trauma patients with severe pelvic fracture

a

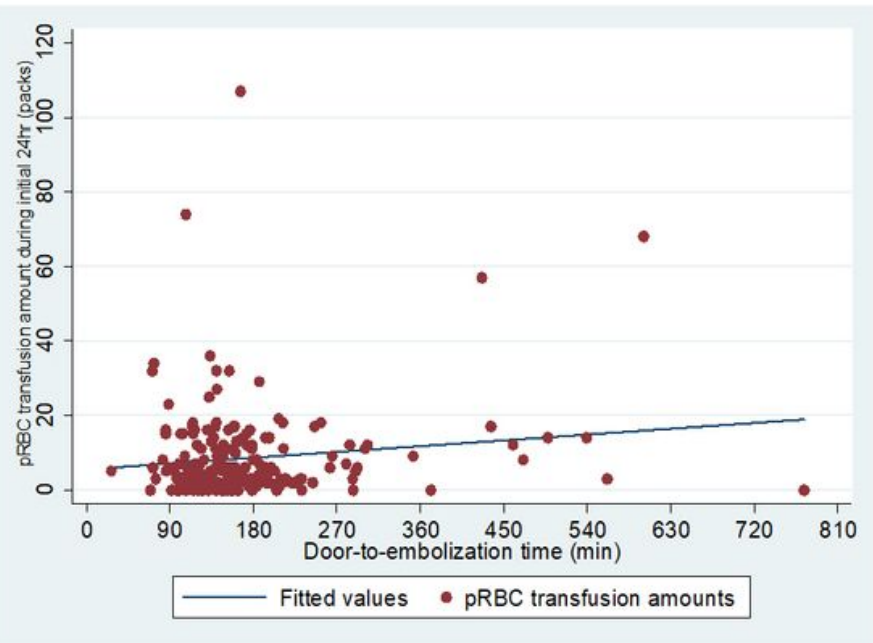

b

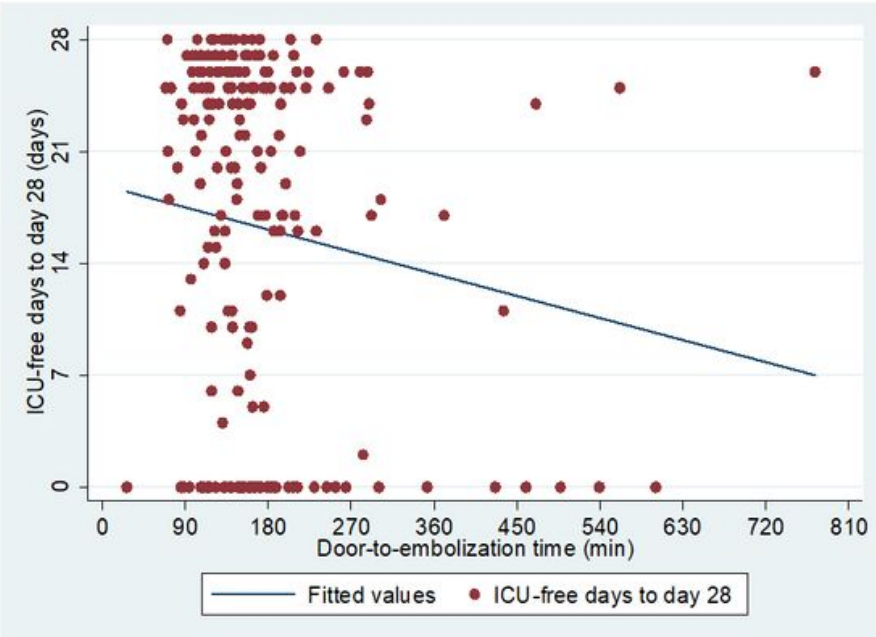

Figure 4

The relationships between (a) door-to-embolization time and the requirement for pRBC transfusion requirement in the initial 24 h, and (b) door-to-embolization time and ICU-free days to day 28

a

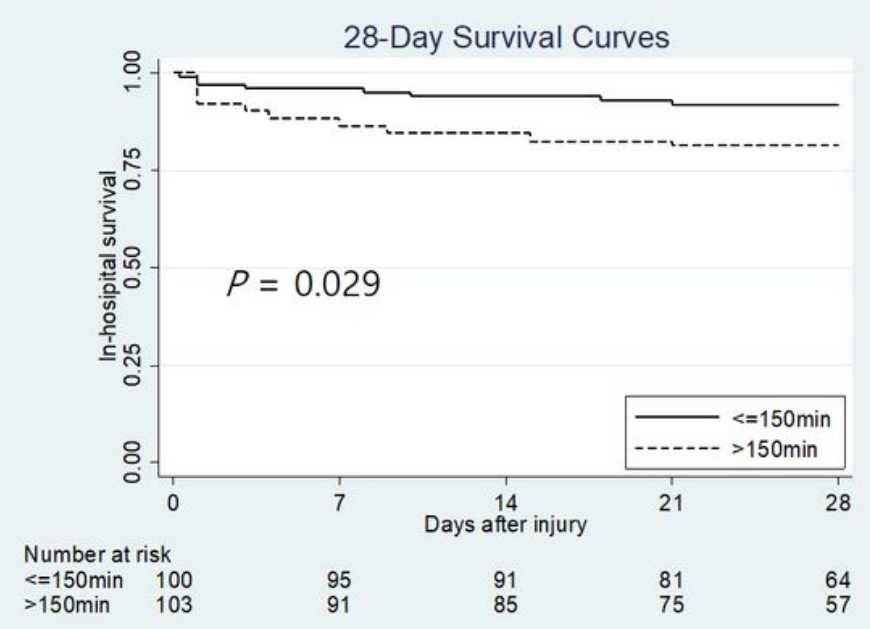

b

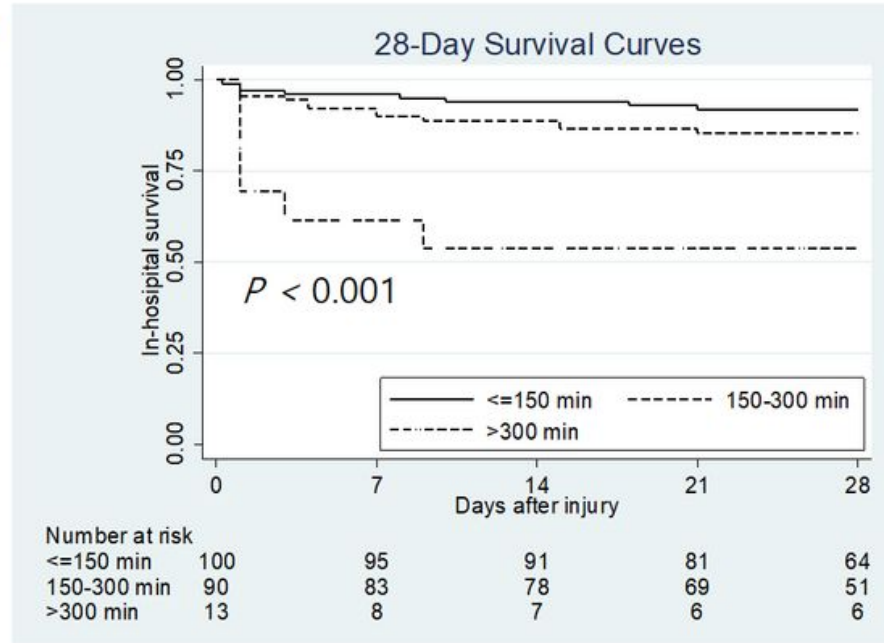

Figure 5

Kaplan-Meier 28-day mortality curves of patients treated with transarterial embolization according to door-toembolization time 